\title{
Pekeris waveguide comparisons of methods for predicting acoustic field amplitude uncertainty caused by a spatially uniform environmental uncertainty $(L)^{\text {a) }}$
}

\author{
Kevin R. James and David R. Dowling ${ }^{\text {b) }}$ \\ Department of Mechanical Engineering, University of Michigan, Ann Arbor, Michigan 48109-2133
}

(Received 24 June 2010; revised 12 November 2010; accepted 23 November 2010)

\begin{abstract}
Acoustic field calculations in underwater environments are often uncertain because the environmental parameters required for such calculations are uncertain. This letter compares the accuracy of direct simulations, the field shifting approximation, and polynomial chaos expansions for predicting acoustic amplitude uncertainty in 100-m-deep Pekeris waveguides having spatially uniform uncertain watercolumn sound speed. When this sound speed is Gaussian-distributed with a standard deviation of $1 \mathrm{~m} / \mathrm{s}$, direct simulations and polynomial chaos expansions, based on 21 field calculations, are more accurate than the field shifting approximation, based on two field calculations. This ranking reverses as the sound-speed standard deviation increases to $20 \mathrm{~m} / \mathrm{s}$. C 2011 Acoustical Society of America.
\end{abstract}

[DOI: $10.1121 / 1.3531814]$

PACS number(s): 43.60.Cg, 43.30.Bp [RAS]

Pages: 589-592

\section{INTRODUCTION}

Uncertainty in environmental parameters is often the dominant source of uncertainty in an underwater acoustic field calculation. In addition, the relationship between environmental parameters and the final field prediction may be highly nonlinear, and explicit determination of the resulting field uncertainty may be computationally prohibitive for real-time applications. Thus, efficient techniques that accurately predict the calculated-field uncertainty arising from environmental-parameter uncertainty are sought for practical applications of active and passive sonar including environmental inversion (Livingston et al., 2006).

In this letter, results are compared from three acoustic uncertainty-prediction methods that are capable of handling the nonlinear sensitivity of the acoustic field to environmental-parameter uncertainty. In particular, the reported comparisons are for predictions of the probability density function (PDF) of acoustic field amplitude, $A$, resulting from a harmonic point source in Pekeris waveguides having a single spatially uniform uncertain environmental parameter, the watercolumn sound speed, $c_{1}$. Field uncertainties associated with phase (or travel time) are not addressed. Instead, this investigation compared amplitude-uncertainty techniques that do not inherently involve averaging but might approach the computational efficiency of mode-based uncertainty estimation (Zingarelli, 2008), a technique that only requires a single field calculation but does involve range, depth, or frequency averaging. Here, the number of acoustic field calculations for each technique is assumed to set its computational burden.

The first method considered here, direct simulation (DS), involves repeatedly calculating the acoustic field at $N_{\mathrm{DS}}$ different equally spaced sound speeds spanning \pm 3 standard

\footnotetext{
a) Based on the work presented at the 157 th meeting of the Acoustical Society of America, Portland, Oregon, May 2009.

b) Author to whom correspondence should be addressed. Electronic mail: drd@umich.edu
}

deviations $\left(\sigma_{c}\right)$ of $c_{1}$ and then linearly interpolating between these calculations, as appropriate, to convert $\operatorname{PDF}\left(c_{1}\right)$ into $\operatorname{PDF}(A)$. When $N_{\mathrm{DS}} \rightarrow \infty$, this method converges to the correct $\operatorname{PDF}(A)$ and it should be considered the default or benchmark approach to acoustic uncertainty prediction. When applied to $M$ uncertain parameters, the computational effort of the simplest implementation of DS increases like $\left(N_{\mathrm{DS}}\right)^{M}$. Monte-Carlo, efficient, or other sparse sampling techniques (not employed here) may reduce this load. In the various comparisons presented here, the DS results are produced from $N_{\text {DS }}=21$ field calculations, a number high enough to produce excellent results at short ranges and low frequencies, but low enough to allow obvious imperfections to arise at longer ranges and higher frequencies.

The second method, field shifting (FS), is approximate and is based on extending the waveguide invariant concept to uncertain environmental parameters (James and Dowling, 2008). Here parametric uncertainties in $c_{1}$ are mapped into spatial shifts, and computed field amplitudes at shifted locations are used to construct an estimated $\operatorname{PDF}(A)$. When applied to $M$ uncertain parameters, the computational effort of FS scales as $M+1$. Thus, all the FS results presented here are based on $N_{\mathrm{FS}}=2$ field calculations.

The third method involves polynomial chaos expansions (PCE), a general approach for representing stochastic processes and fields that has recently been applied to underwater acoustic uncertainty assessment. In the present study, it involved summing a series of $Q$ basis functions whose range-, depth-, and frequency-dependent coefficients were determined from the solution of a system of $Q$ coupled partial differential equations. This technique converges to the correct $\operatorname{PDF}(A)$ when $Q \rightarrow \infty$, and modest $Q(<10)$ may produce reliable results when the uncertain environmental parameter or parameters have finite correlation lengths. The PCE solution method employed here follows that in Finette (2006) but is extended to the full Helmholtz equation and penetrable ocean bottoms with a simplification to disregard phase. The computational burden of this particular solution 
technique is equivalent to $Q$ field calculations, so $Q$ is set to 21 for computational-burden parity with the DS calculations. Application of PCE to multiple uncertain variables requires a different solution technique, where $Q$ cannot be equated with an equivalent number of field calculations. Ongoing research in PCE methods (Finette, 2009) may yield a lower computational burden than DS for multiple uncertain variables with finite correlation lengths.

\section{COMPARISON SCOPE}

The accuracy of these three methods for estimating $\operatorname{PDF}(A)$ are compared in Pekeris waveguide environments (Fig. 1) with a Gaussian-distributed spatially uniform watercolumn sound speed having a mean of $\bar{c}_{1}=1500 \mathrm{~m} / \mathrm{s}$ and a standard deviation $\sigma_{c}$ that set to $1,3,5,10,15$, and $20 \mathrm{~m} / \mathrm{s}$. This ideal underwater environment was chosen for this comparison study because it supports multipath sound propagation but is simple enough for direct $\operatorname{PDF}(A)$ determination using all three techniques at significant sound-speed uncertainty levels. Although all three methods may be applied in more realistic environments, the relative dearth of PCE solutions for wave propagation problems limited the scope of this study. For the DS and FS methods the requisite acoustic field calculations were completed via a traditional modal sum as described in Jensen et al. (1994).

For the present study, point-to-point uncertainty calculations were completed for 900 different propagation scenarios in a 100-m-deep sound channel with a harmonic point source placed at $40 \mathrm{~m}$ depth. The various scenarios included ten acoustic frequencies from $100 \mathrm{~Hz}$ to $1 \mathrm{kHz}$ (integer multiples of $100 \mathrm{~Hz}$ ), ten source-receiver ranges from 1 to $10 \mathrm{~km}$ (integer multiples of $1 \mathrm{~km})$, three receiver depths $(25,50$, and 80 $\mathrm{m})$, and three nominal bottom types (silt, sand, and gravel). Appropriate bottom density, sound speed, and absorption values were taken from Table 1.3 in Jensen et al. (1994).

PDF accuracy was assessed from an $L_{1}$ error norm determined from the approximate distribution of interest, $\operatorname{PDF}_{a}(A)$ and a numerically converged reference distribution,

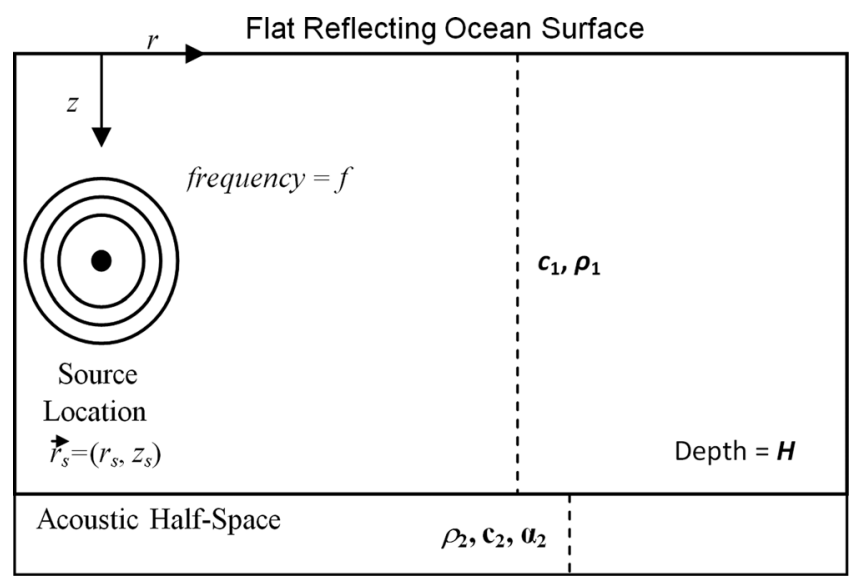

FIG. 1. The Pekeris waveguide. The environmental parameters are the water-column sound speed and density $\left(c_{1}\right.$ and $\left.\rho_{1}\right)$, the bottom sound speed, density, and absorptivity $\left(\mathrm{c}_{2}, \rho_{2}\right.$, and $\left.\alpha_{2}\right)$, and the channel depth $H=100 \mathrm{~m}$. The acoustic point source is specified by its frequency $f$ and depth $z_{s}=40 \mathrm{~m}$. Here, $c_{1}$ is an uncertain parameter, and $c_{2}, \rho_{2}, \alpha_{2}, r, z$, and $f$ are varied to produce a variety of propagation scenarios.
$\operatorname{PDF}_{r}(A)$, constructed from the DS technique and 401 field calculations.

$$
L_{1}=\int_{o}^{\infty}\left|\mathrm{PDF}_{a}(A)-\mathrm{PDF}_{r}(A)\right| d A
$$

The $L_{1}$ error norm was chosen for this study because it is simple and dimensionless. The $L_{1}$ error norm is bounded, $0 \leq L_{1}$ $\leq 2$, with lower values of $L_{1}$ indicating higher $\mathrm{PDF}_{a}$ accuracy. A value of $L_{1} \approx 0.5$ typically indicates errors of less than one or two decibels in the mean and variance of $A$, and thereby provides a nominal engineering-accuracy cutoff above which a predicted $\mathrm{PDF}_{a}(A)$ might be considered unacceptable.

\section{RESULTS}

Sample results for the three techniques for the 900 propagation scenarios are shown as scatter plots in Fig. 2 for $\sigma_{c}=10 \mathrm{~m} / \mathrm{s}$. For nearly all scenarios, this sound-speed uncertainty leads to a nonlinear field amplitude dependence on sound speed, and the resulting $\operatorname{PDF}(A)$ was typically spread over an amplitude range of $10 \mathrm{~dB}$ or more. The empirical dimensionless parameter $\eta=\left(\omega r / \bar{c}_{1}\right)\left(c_{2} / \bar{c}_{1}\right)$ (where $c_{1}$ and $c_{2}$ are the sound speeds in the water column and bottom, respectively) on the horizontal axis in each panel of Fig. 2 indicates the sound channel's contribution to the severity of this nonlinearity. In general, higher frequencies, longer ranges, and faster bottoms - all leading to higher $\eta$-provided greater challenges to the three uncertainty-prediction techniques. The dashed horizontal line in each panel lies at $L_{1}=0.5$.

Figure 2(a) shows $L_{1}$ results for the DS method when $N_{\text {DS }}=21$. Here, $95 \%$ of the $L_{1}$ values fall below 0.43 , and there is a general trend of increasing $L_{1}$ with increasing $\eta$. This trend occurs because 21 field amplitude samples tend to yield a progressively less accurate determination of $\operatorname{PDF}(A)$ as the amplitude sensitivity to variations in $c_{1}$ becomes increasingly nonlinear.

Figure 2(b) shows $L_{1}$ results for the FS method when $N_{\mathrm{FS}}=2$. Here, $95 \%$ of the FS $L_{1}$ values fall below 0.40 , and $50 \%$ of the FS $L_{1}$ values are lower than their DS counter parts. Thus, as implemented here, these two techniques provide comparable accuracy at a coarse statistical level but differ by an order of magnitude in their computational burden. Interestingly, the sweep of FS- $L_{1}$ data is nearly horizontal and shows that the accuracy of the FS method is relatively independent of the sound channel's propagation complexity as represented by $\eta$. The accuracy of FS is dependent on the applicability of the spatial-shifting assumption to the computed acoustic fields, and in this sound channel, increasing $\eta$ does not significantly decrease the accuracy of this assumption.

Figure 2(c) shows $L_{1}$ results for the PCE method when $Q=21$. Here, $95 \%$ of the $L_{1}$ values fall below 0.70 , and there is a general trend of increasing $L_{1}$ with increasing $\eta$, similar to the DS results in Fig. 2(a). However, the $L_{1}$ errors tend to be larger for PCE with $Q=21$ compared to DS with $N_{\mathrm{DS}}=21$. The primary performance limitation of PCE in these tests is set by this series-truncation value $Q=21$. A higher $Q$ would decrease PCE $L_{1}$ values. 


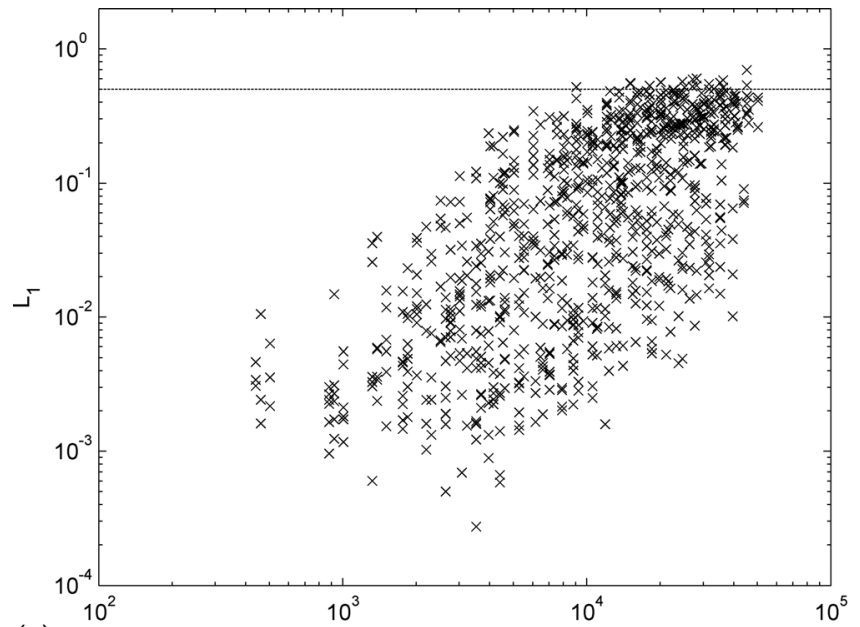

(a)

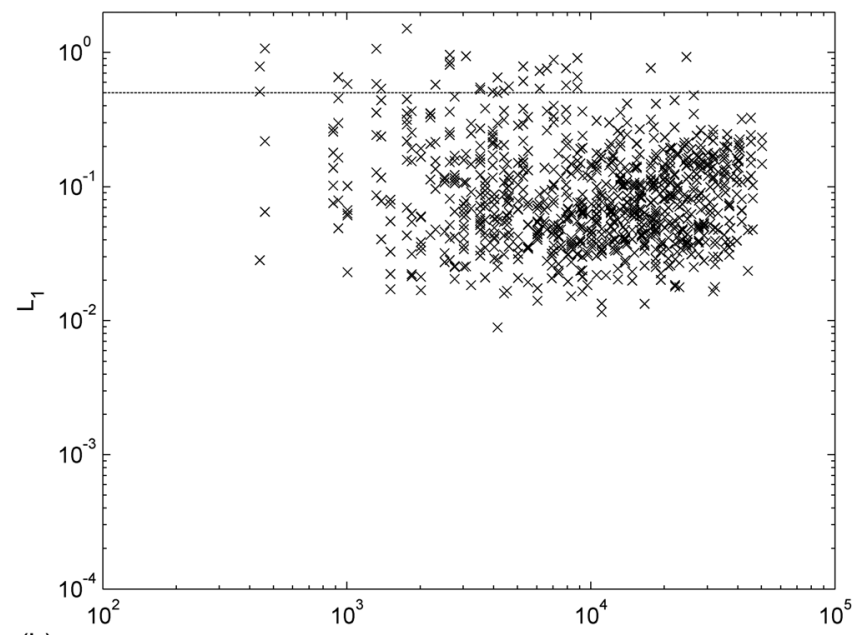

(b)

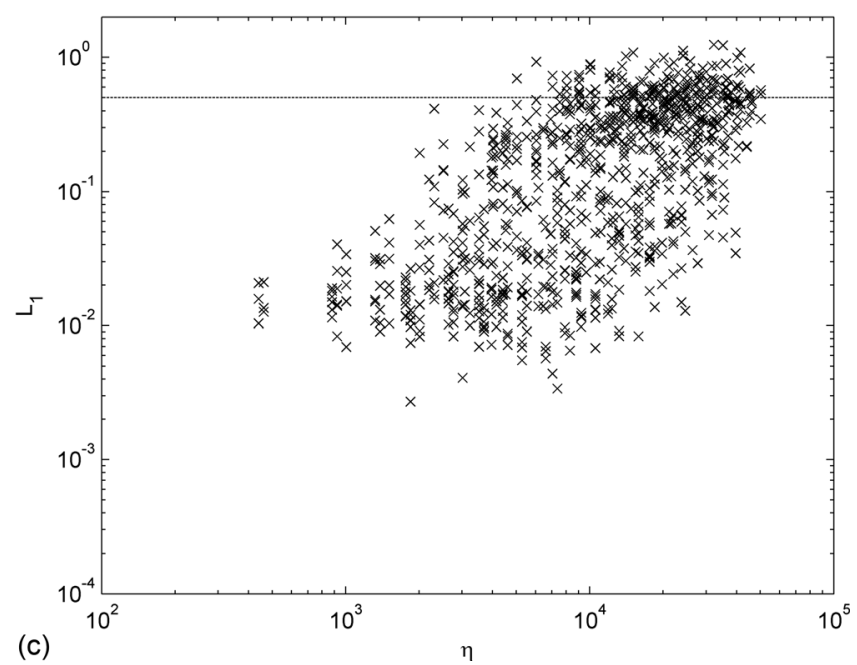

(c)

FIG. 2. (a) $L_{1}$ values for the DS method based on 21 field calculations vs $\eta=\left(\omega r / \bar{c}_{1}\right)\left(c_{2} / \bar{c}_{1}\right)$, for 900 different propagation scenarios with a water-column sound-speed standard deviation of $10 \mathrm{~m} / \mathrm{s}$. The dashed line corresponds to an $L_{1}$ of 0.5 . The reference PDF in each case was determined from DS using 401 field calculations. (b) Same as (a), except that the results are from the FS method. (c) Same as (a), except that the results are from the PCE method.

An overall performance comparison, based on more than $16000 \operatorname{PDF}(A)$-accuracy assessments, is presented in Fig. 3 where $L_{1}$ statistics are plotted for all three techniques for $\sigma_{c}=1,3,5,10,15$, and $20 \mathrm{~m} / \mathrm{s}$. Here the results from the

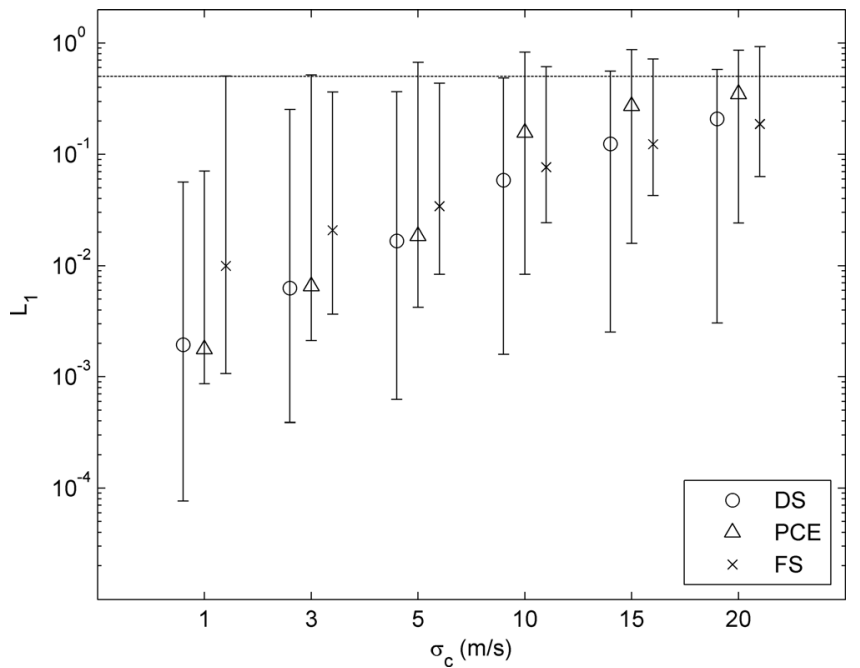

FIG. 3. $L_{1}$ vs water-column sound-speed standard deviation $\sigma_{c}=1,3,5$, 10,15 , and $20 \mathrm{~m} / \mathrm{s}$ for all 900 propagation scenarios. Median $L_{1}$ values are represented by the symbols (circles for DS with $N_{\mathrm{DS}}=21$, triangles for PCE with $Q=21$, and crosses for FS with $N_{\mathrm{FS}}=2$ ). The error bars span the $95 \%$ confidence interval. For clarity, results from the various techniques are shifted slightly at each of the six values of $\sigma_{c}$.

900 propagation scenarios are condensed to median $L_{1}$ values represented by symbols (circles for DS with $N_{\mathrm{DS}}=21$, triangles for PCE with $Q=21$, and crosses for FS with $N_{\mathrm{FS}}=2$ ) with error bars that span the $95 \%$ confidence interval of the $L_{1}$ results. Although the median $L_{1}$ increases monotonically with increasing $\sigma_{c}$ for all three techniques, it does so at different rates. For $\sigma_{c}=1,3$, and $5 \mathrm{~m} / \mathrm{s}$, the DS and PCE methods produce lower median $L_{1}$ errors than FS, but these differences may not be significant if the $L_{1}$-error tolerance is 0.5 since the median $L_{1}$ errors for all three technique are well below this value. Interestingly, the FS method's median $L_{1}$ error is lowest for $\sigma_{c}=15$ and $20 \mathrm{~m} / \mathrm{s}$. Here, the median- $L_{1}$-error crossover point is specific to $N_{\mathrm{DS}}=Q=21$ since $L_{1}$ values from the DS and PCE methods would be smaller for larger $N_{\mathrm{DS}}$ and $Q$. However, in applications requiring real-time acoustic-uncertainty predictions, suppressing $L_{1}$-errors below 0.1 may not be as important as computational efficiency.

\section{CONCLUSIONS}

The FS approximation may be a computationally efficient alternative to the DS and PCE methods for predicting acoustic field amplitude uncertainties caused by spatially uniform uncertain environmental parameters when acoustic field calculations dominate the computational effort. DS or PCE methods are superior when computational effort is not an issue. The results presented here illustrate how accuracy scales with sound channel complexity for each technique. For low soundspeed uncertainties, low frequencies, slow bottoms, and short ranges, the sensitivity of field amplitude to sound-speed changes at a fixed field point may be nearly parabolic, or even linear, and may be well approximated by linear or polynomial fits. As these acoustic and environmental parameters increase and amplitude-sound-speed sensitivity curves become more complicated, more samples are required to maintain a similar level of accuracy with the DS and PCE techniques. The 
accuracy of FS, however, does not explicitly depend on these acoustic and environmental parameters. Instead its accuracy depends on the spatial structure of the field and the validity of the FS assumptions in a particular sound channel.

\section{ACKNOWLEDGMENT}

This research effort was sponsored by the Office of Naval Research, award reference number N00014-05-1-0243.

Finette, S. (2006). "A stochastic representation of environmental uncertainty and its coupling to acoustic wave propagation in ocean waveguides," J. Acoust. Soc. Am. 120, 2567-2579.
Finette, S. (2009). "A stochastic response surface formulation of acoustic propagation through an uncertain ocean waveguide environment," J. Acoust. Soc. Am. 126, 2242-2247.

James, K. R., and Dowling, D. R. (2008). "A method for approximating acoustic-field-amplitude uncertainty caused by environmental uncertainties," J. Acoust. Soc. Am. 124, 1465-1476.

Jensen, F., Kuperman, W., Porter, M., and Schmidt, H. (1994). Computational Ocean Acoustics (AIP, New York), 41, pp. 121-126.

Livingston, E. S., Goff, J. A., Finette, S., Abbot, P., Lynch, J. F., and Hodgkiss, W. S. (2006). "Guest editorial capturing uncertainty in the tactical ocean environment," IEEE J. Ocean. Eng. 31, 245-248.

Zingarelli, R. A. (2008). "A mode-based technique for estimating uncertainty in range-averaged transmission loss results for underwater acoustic calculations,” J. Acoust. Soc. Am. 124, EL218-EL222. 